\title{
Multi-Robot Localization Using Relative Observations
}

\author{
Agostino Martinelli, Frederic Pont and Roland Siegwart \\ Swiss Federal Institute of Technology \\ Lausanne CH-1015, Switzerland \\ agostino.martinelli, frederic.pont, roland.siegwart @epfl.ch
}

\begin{abstract}
In this paper we consider the problem of simultaneously localizing all members of a team of robots. Each robot is equipped with proprioceptive sensors and exteroceptive sensors. The latter provide relative observations between the robots. Proprioceptive and exteroceptive data are fused with an Extended Kalman Filter. We derive the equations for this estimator for the most general relative observation between two robots. Then we consider three special cases of relative observations and we present the structure of the filter for each case. Finally, we study the performance of the approach through many accurate simulations.
\end{abstract}

Index Terms-Robot Navigation, Kalman filter, Multi-Robot Localization, Sensor Fusion, Relative Observation

\section{INTRODUCTION}

In most cases, autonomous mobile robots are required to know precisely their position and orientation in order to successfully perform their mission. This is usually achieved by fusing proprioceptive data (gathered by sensors monitoring the motion of the vehicle, like encoders) with exteroceptive data (e.g. [1], [3], [5], [12]).

Most of the localization methods have been developed for applications involving a single robot. Current research investigates applications where a team of robots collaborates to fulfill a mission. Single-robot localization approaches are not optimized to estimate the positions of all members of a team of collaborating robots. Indeed, an optimal strategy must take advantage of relative observations (detection of other robots).

Fox and collaborators [7] introduced a probabilistic approach based on Markov localization. Their approach has been validated through real experiments showing a drastic improvement in localization speed and accuracy when compared to conventional single robot localization. Other approaches take advantage of relative observations for multi-robot localization [8], [9], [10], [13], [14], [15]. In [9] a method based on a combination of maximum likelihood estimation and numerical optimization was introduced. This method allows to reduce the error in the robot localization by using the information coming from relative observations among the robots in the team. In [14], an Extended Kalman Filter (EKF) is used to fuse proprioceptive and exteroceptive sensor data. The equations of this filter are written in a decentralized form, allowing the decomposition into a number of smaller communicating filters. The approach relies on a particular relative observation, that is the relative configuration (position and orientation) between two robots. Experiments with a group of three robots successfully validated the method.

In this paper we extend the EKF approach introduced in [14] by considering the most general relative observation between two robots. The interest of this extension relies on the fact that in many real applications the robots can be equipped with exteroceptive sensors not able to provide the special relative observation considered in [14]. In order to exploit the information contained in any relative observation between two robots, we derive (section II) the $E K F$ equations to integrate a generic relative observation. Then, three different relative observations are considered, that is relative bearing, relative distance and relative orientation. The structure of the filter is explicitly derived in section III. In section IV the results from several accurate simulations for the previous three relative observations are provided. Finally, some conclusions and future research are presented in section $\mathrm{V}$.

\section{LOCALIZATION}

The goal of this section is to present the adopted strategy to estimate the configuration of each robot (from now on $R_{i}$ ). Each $R_{i}$ is equipped with encoders and an exteroceptive sensor. The latter is able to detect and identify other robots, therefore providing relative observation.

Let us indicate the configuration of $R_{i}$ with $X_{i}=$ $\left[x_{i}, y_{i}, \theta_{i}\right]^{T}$. The state we want to estimate is then

$$
X=\left[X_{1}, X_{2}, X_{3}, \ldots, X_{N}\right]^{T}
$$

We use an Extended Kalman Filter (EKF) to fuse encoder data and the data from the exteroceptive sensor. Therefore, this EKF will update the state in (1) and its covariance matrix, which is:

$$
P=\left[\begin{array}{cccc}
P_{11} & P_{12} & \ldots & P_{1 N} \\
P_{21} & P_{22} & \ldots & P_{2 N} \\
\ldots & \ldots & \ldots & \ldots \\
P_{N 1} & P_{N 2} & \ldots & P_{N N}
\end{array}\right]
$$

\section{A. Encoder Data Integration}

The dynamics for the $R_{i}$ can be modelled through the following equation:

$$
X_{i_{k+1}}=f\left(X_{i_{k}}, u_{i_{k}}\right)
$$


where $k$ is the time and $u_{i_{k}}$ are the wheel displacements between the time $k$ and $k+1$ which are directly evaluated by the encoders $\left(u_{i_{k}}^{e}\right)$. Clearly, this estimation is affected by an error. We assume the following encoder error model:

$$
u_{i_{k}}=N\left(u_{i_{k}}^{e}, Q_{i_{k}}\right)
$$

We assumed that the function $f$ in (3) is the same for all robots (in other words the drive system is the same).

The equations to update the state in (1) and its covariance in (2) are [2]:

$$
\begin{gathered}
\hat{X}_{i_{k+1}}=f\left(\hat{X}_{i_{k}}, u_{i_{k}}^{e}\right) \\
P_{i i_{k+1}}=F_{x_{i_{k}}} P_{i i_{k}} F_{x_{i_{k}}}^{T}+F_{u_{i_{k}}} Q_{i_{k}} F_{u_{i_{k}}}^{T} \\
P_{i j_{k+1}}=F_{x_{i_{k}}} P_{i j_{k}} F_{x_{j_{k}}}^{T}
\end{gathered}
$$

where $\hat{X}_{i_{k}}$ is the estimated $X_{i_{k}}$ at the time $k, F_{x_{i_{k}}}$ and $F_{u_{i_{k}}}$ are respectively the Jacobian of the function $f$ with respect to the state $X_{i}$ and $u_{i}$ in $\left(\hat{X}_{i_{k}}, u_{i_{k}}^{e}\right)$.

\section{B. Exteroceptive Sensor Data Integration}

Let us suppose that at a given time $R_{i}$ observes $R_{j}$ through its exteroceptive sensor. We model this relative observation through the following equation:

$$
z=h\left(X_{i}, X_{j}\right)+w
$$

We assumed that the function $h$ is the same for all the relative observations. $w$ is an error with Gaussian distribution, zero mean value, and covariance matrix $R$.

By applying the Kalman filter equations (see the appendix) we can update the new state and the new covariance matrix. We obtain for $R_{l}$

$$
\begin{gathered}
\hat{X}_{l_{\text {new }}}=\hat{X}_{l}+ \\
+\left(P_{l i} H_{i}^{T}+P_{l j} H_{j}^{T}\right) P_{z z}^{-1}\left(z-h\left(\hat{X}_{i}, \hat{X}_{j}\right)\right)
\end{gathered}
$$

where $P_{z z}$ is the covariance of the innovation $\left(z-h\left(\hat{X}_{i}, \hat{X}_{j}\right)\right)$ computed in the appendix and $H_{i}$ and $H_{j}$ are respectively the Jacobian of the function $h$ with respect to $X_{i}$ and $X_{j}$ both computed in $\left(\hat{X}_{i}, \hat{X}_{j}\right)$.

We obtain (see the appendix) the following equation for the update of the component $P_{l f}$ in the covariance matrix

$$
\begin{gathered}
P_{l f_{n e w}}=P_{l f}+ \\
-\left(P_{l i} H_{i}^{T}+P_{l j} H_{j}^{T}\right) P_{z z}^{-1}\left(H_{i} P_{i f}+H_{j} P_{j f}\right)
\end{gathered}
$$

As shown in [14], communication among the robots is required only when a relative observation occurs. This allows for implementation on real robots with limited communication bandwidth. Furthermore, the global localization error is not bounded. Indeed, the Observability matrix is not full rank. To have a bound it is necessary that at least one robot has absolute positioning capabilities.

\section{RELATIVE OBSERVATION}

In the relative observation one robot detects and identifies another one and measures some relative quantity through its exteroceptive sensors. This quantity will depend on both robot configurations. We will call the first robot the Observer and the latter the Observed. In this section we consider three special cases of relative observations (see figure 1):

- relative bearing (i.e. direction of the Observed in the reference of the Observer);

- relative distance;

- relative orientation (i.e. the orientation of the Observed in the reference of the Observer)

The relative observation is completely defined through the function $h$ appearing in equation (8). Therefore, in order to derive the structure of the filter for the three mentioned cases, it is necessary to specify the analytical expression of $h$, to compute its Jacobian and to apply the equations derived in the previous section (in particular, the equations (9) and (10)).

The previous three observations are chosen because of the following two reasons:

- when the three observations are simultaneously combined together they contain all the necessary information to estimate the configuration of the Observed in the reference of the Observer (this global observation is the one considered in [14]);

- they can be easily implemented on real platforms with good accuracy (in particular, for bearing, a camera can be used).

Let us derive the equations for the three relative observations. The required analytical expressions for function $h$ in (8) can be obtained from fig. 1.

\section{A. Relative Bearing}

It is possible to express the bearing angle in terms of the two robot configurations through the following expression:

$$
\begin{gathered}
z_{b}=h_{b}\left(X_{i}, X_{j}\right)= \\
=\tan ^{-1}\left(\frac{-\sin \theta_{i} \Delta x+\cos \theta_{i} \Delta y}{\cos \theta_{i} \Delta x+\sin \theta_{i} \Delta y}\right)
\end{gathered}
$$

where

$$
\Delta x=x_{j}-x_{i} ; \quad \Delta y=y_{j}-y_{i}
$$

The two Jacobian $H_{i}$ and $H_{j}$ in the update equations (9) and (10), are obtained from the expression in (11):

$$
\begin{gathered}
H_{i}^{b}=\left[\frac{\Delta y}{\Delta x^{2}+\Delta y^{2}}, \frac{-\Delta x}{\Delta x^{2}+\Delta y^{2}},-1\right] \\
H_{j}^{b}=\left[\begin{array}{lll}
\frac{-\Delta y}{\Delta x^{2}+\Delta y^{2}}, & \frac{\Delta x}{\Delta x^{2}+\Delta y^{2}}, & 0
\end{array}\right]
\end{gathered}
$$




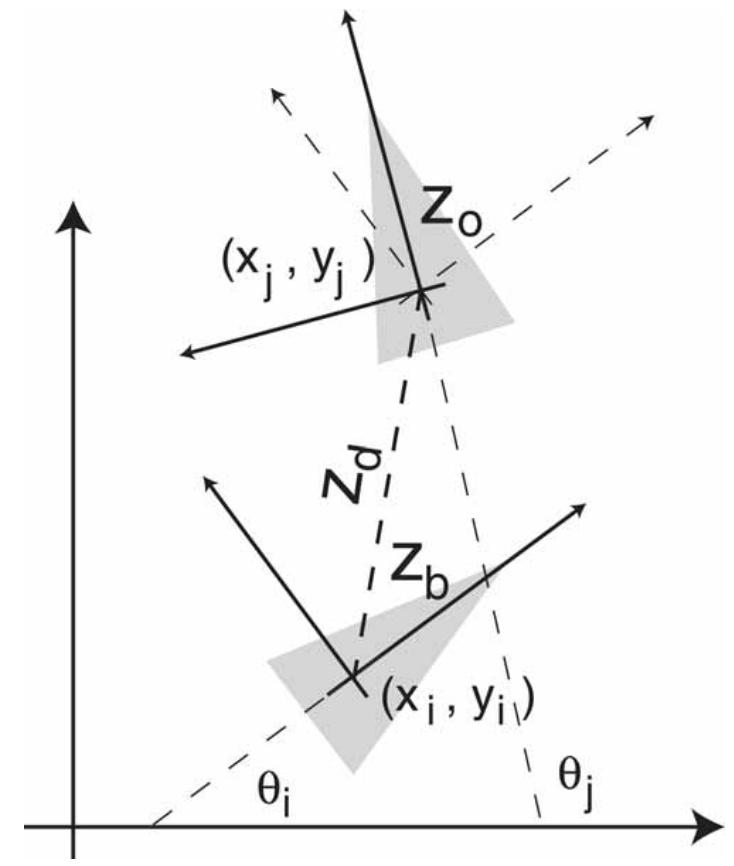

Fig. 1. Relative observations between two robots. $z_{b}$ is the bearing, $z_{d}$ the relative distance and $z_{o}$ the relative orientation

\section{B. Relative Distance}

The expression for the relative distance is:

$$
z_{d}=h_{d}\left(X_{i}, X_{j}\right)=\sqrt{\Delta x^{2}+\Delta y^{2}}
$$

and for the two Jacobian we get:

$$
\begin{aligned}
& H_{i}^{d}=\left[\begin{array}{lll}
\frac{-\Delta x}{\sqrt{\Delta x^{2}+\Delta y^{2}}}, & \frac{-\Delta y}{\sqrt{\Delta x^{2}+\Delta y^{2}}}, & 0
\end{array}\right] \\
& H_{j}^{d}=\left[\begin{array}{lll}
\frac{\Delta x}{\sqrt{\Delta x^{2}+\Delta y^{2}}}, & \frac{\Delta y}{\sqrt{\Delta x^{2}+\Delta y^{2}}}, & 0
\end{array}\right]
\end{aligned}
$$

\section{Relative Orientation}

The expression for the relative orientation is:

$$
z_{o}=h_{o}\left(X_{i}, X_{j}\right)=\theta_{j}-\theta_{i}
$$

and the two Jacobian are:

$$
H_{i}^{o}=\left[\begin{array}{lll}
0, & 0, & -1
\end{array}\right], \quad H_{j}^{o}=\left[\begin{array}{lll}
0, & 0, & 1
\end{array}\right]
$$

\section{Results And Discussion}

We validated the proposed approach through several accurate simulations where we considered the three relative observations introduced and also observations consisting of a combination of them.

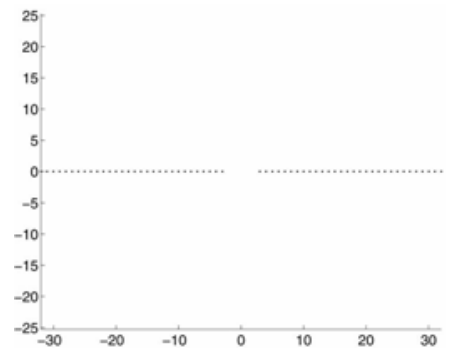

(a)

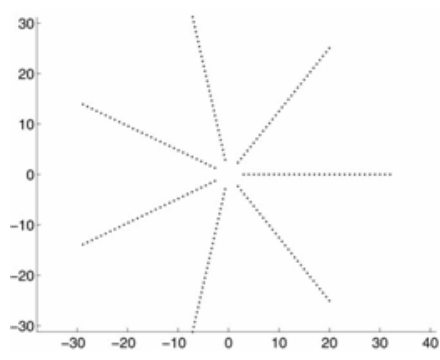

(b)
Fig. 2. The simulated robot motions for the experiment with 2 (a) and 7 (b) robots.

\section{A. Simulated Environment}

We simulated several experiments by varying the number of robots from two to seven. We also considered different robot trajectories. In particular, we moved the robot along straight lines, i.e. we set $v_{R}=v_{L}$ for each robot (see fig 2), straight lines and pure rotations (i.e. $v_{R}=v_{L}$ for some robots and $v_{R}=-v_{L}$ for the other ones) and more general trajectories obtained by setting at each time step the value of $v_{R}$ and $v_{L}$ randomly.

In each experiment data coming from the encoder sensors were delivered at the frequency of $100 \mathrm{~Hz}$, in agreement with the experiments carried out on real platforms in our laboratory (e.g. [1]). The simulated robots are equipped with a differential drive system. We adopted the same odometry error model introduced in [4] where the actual translation of the right and left wheel at a given time step is assumed to be a gaussian random variable satisfying the following relation:

$$
\begin{gathered}
\delta \rho^{R / L}=\overline{\delta \rho}^{R / L}+\nu^{R / L} \\
\overline{\delta \rho}^{R / L}=\delta \rho^{e R / L} \delta_{R / L} \\
\nu^{R / L} \sim N\left(0, K_{R / L}\left|\delta \rho^{e R / L}\right|\right)
\end{gathered}
$$

In other words, both $\delta \rho^{R}$ and $\delta \rho^{L}$ are assumed to be gaussian random variables, whose mean values are given by the encoder readings (respectively $\delta \rho^{e R}$ and $\delta \rho^{e L}$ ) corrected for the systematic errors (which are assumed to increase linearly with the distance travelled by each wheel), and whose variances also increase linearly with the travelled distance. Furthermore, it is assumed that $\delta \rho^{R}$ and $\delta \rho^{L}$ are uncorrelated. In our simulation we adopted $\delta_{R}=\delta_{L}=1$ (i.e. encoders perfectly calibrated) and $K_{R}=K_{L}=510^{-5} \mathrm{~m}$ (that is the value experimentally estimated for our robot in our laboratory, [11]).

We simulated all three relative observations introduced in the previous section. In particular, the frequency for these data was fixed to $1 \mathrm{~Hz}$ which is, in our opinion, reasonably achievable in real experiments. The data consist of all the relative observations among the robots. This point could be questionable, since each robot has to sense all the other 


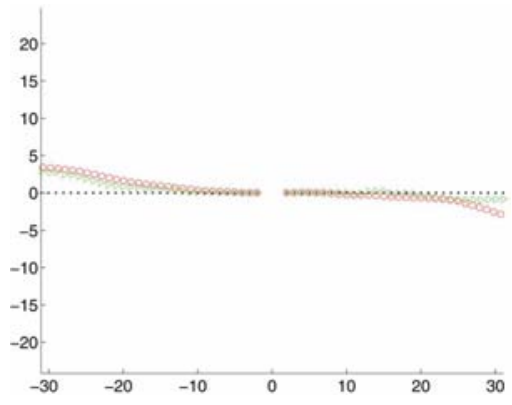

Fig. 3. The experiment with two robots. The real motions (black dotted line) are plotted together with the trajectories estimated by using only odometry (red $o$ ) and by fusing the odometry with the relative distances (green $\triangleright$ ). The unities are $m$ for both axes.

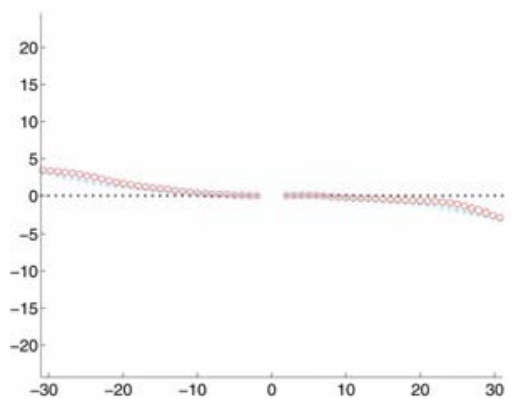

Fig. 4. As in the figure 3 but the sky-blue + is adopted to represent the trajectories estimated by fusing odometry with the relative orientation.

ones. However, in a real experiment with many robots, it is reasonable to think that each member of the team is able to sense at least another one during its navigation. For this reason, the experiment with only two robots could be used to get an upper bound for the error for the cases with more than two robots. Furthermore, in order to overcome this problem, control strategies as the one introduced in [6], could be adopted to move each robot in presence of constraints. This problem can also be reduced by using $360^{\circ}$ sensors, like an omnicam. Regarding the adopted error model for the simulated relative observations, we adopted the following variances, respectively for the case of the relative bearing, relative distance and relative orientation: $\sigma_{B}^{2}=\left(1^{o}\right)^{2}, \sigma_{D}^{2}=$ $(0.01 \mathrm{~m})^{2}$ and $\sigma_{O}^{2}=\left(1^{o}\right)^{2}$. Note that regarding the relative bearing, it is possible to achieve much higher accuracy with an omnicam ([10]).

\section{B. Results}

Figures 3, 4, 5 and 6 illustrate the results for the simulated experiment with two robots moving along the straight lines in fig $2 a$. We conclude that the accuracy on the localization is strongly improved by using the relative bearing. The result obtained by fusing all three relative observations with odometry is slightly better than the one obtained by fusing only the relative bearing with the odometry (in particular the final position error after $30 \mathrm{~m}$ of navigation is $0.0175 \mathrm{~m}$ when all three relative observations are integrated and $0.0356 \mathrm{~m}$ when only the relative bearing is adopted).

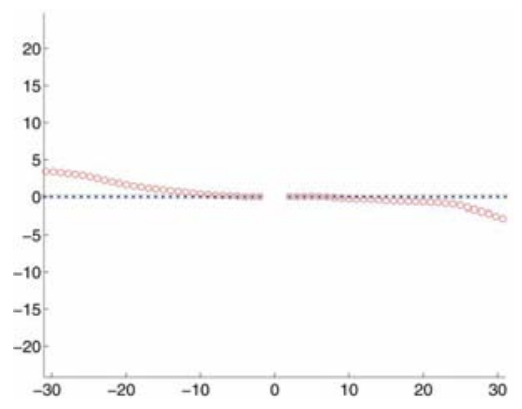

Fig. 5. As in the figure 3 but the blue $\times$ is adopted to represent the trajectories estimated by fusing odometry with the relative bearing.

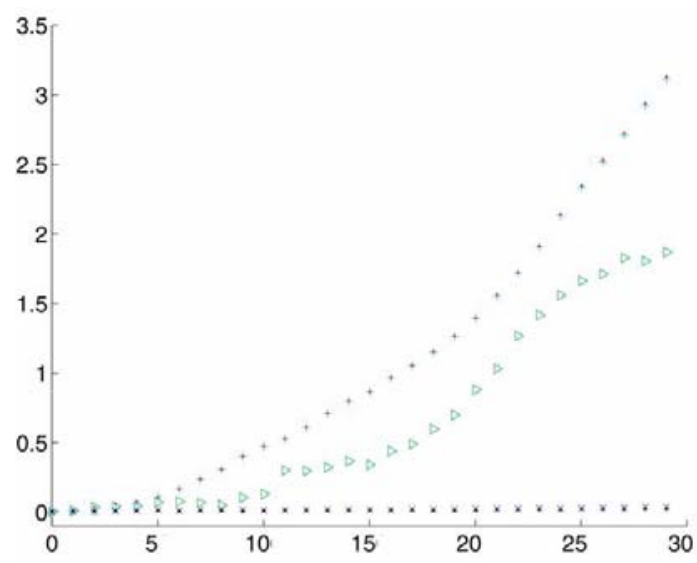

Fig. 6. The error for the estimated position vs the distance travelled by each robot when the estimation is carried out with only odometry (red .), fusion of odometry with the relative distance (green $\triangleright$ ), fusion of odometry with the relative orientation (sky-blue + ), fusion of odometry with the relative bearing (blue $\times$ ) and fusion of odometry with the three relative observations together (black .). The plotted error is averaged over the two robots.

Figures 7, 8, 9 and 10 illustrate the simulation with seven robots moving along the straight lines in fig $2 b$. The results are quite similar to the ones obtained with two robots. The final position error after $30 \mathrm{~m}$ of navigation is shown in table I. The estimation is carried out by using only odometry $\left(1^{\text {st }}\right.$ column), by fusing the odometry with the relative distance ( $2^{\text {nd }}$ column), with the relative orientation ( $3^{\text {rd }}$ column), with the relative bearing ( $4^{\text {st }}$ column) and with all the relative observations ( $5^{\text {st }}$ column)

Regarding other robot trajectories, similar results can be obtained when some robots in the team perform pure rotations (i.e. $v_{R}=-v_{L}$ ) and some other move along straight lines. On the other hand, for more general robot trajectories (obtained by setting at each time step the value of $v_{R}$ and $v_{L}$ randomly), the results can be sometimes different. Indeed, the

\begin{tabular}{|c|c|c|c|c|}
\hline Odometry & Distance & Orientation & Bearing & All \\
\hline 2.7443 & 0.4807 & 1.4098 & 0.0320 & 0.0196 \\
\hline
\end{tabular}

TABLE I

THE FINAL POSITION ERROR WITH SEVEN ROBOTS. 


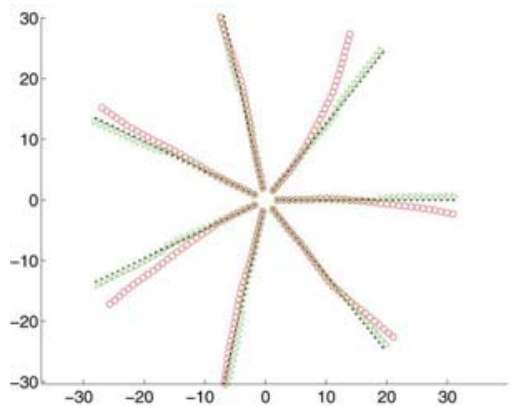

Fig. 7. The experiment with seven robots. The real motions (black dotted line) are plotted together with the trajectories estimated by using only odometry (red $o$ ) and by fusing the odometry with the relative distances (green $\triangleright$ ). The unities are $m$ for both axes.

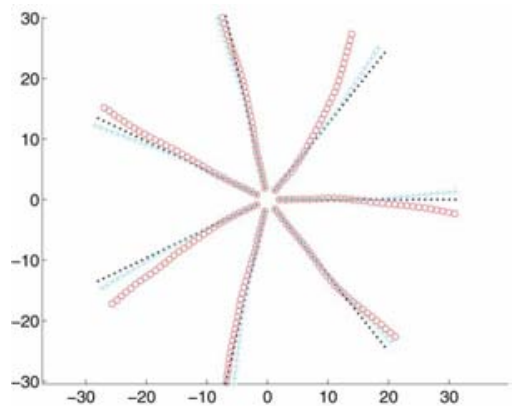

Fig. 8. As in the figure 7 but the sky-blue + is adopted to represent the trajectories estimated by fusing odometry with the relative orientation.

relative bearing usually shows slightly better performances with respect to the other relative observations but it is not always the case. However, simulations tend to indicate that the relative bearing clearly outperforms other relative observations whenever the robot trajectories are similar as the ones presented on figure 2.

\section{CONClusions And Future RESEARCH}

In this paper we considered the problem of localizing simultaneously all members of a team of robots able to sense one another. An Extended Kalman Filter was adopted to estimate a state containing all the configurations of the robots. This filter fuses the data coming from the odometry and the exteroceptive sensors of each robot.

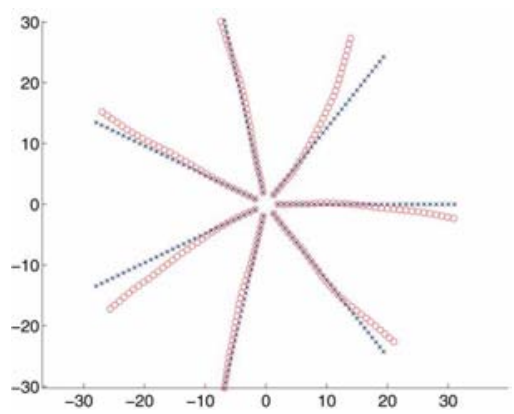

Fig. 9. As in the figure 7 but the blue $\times$ is adopted to represent the trajectories estimated by fusing odometry with the relative bearing.

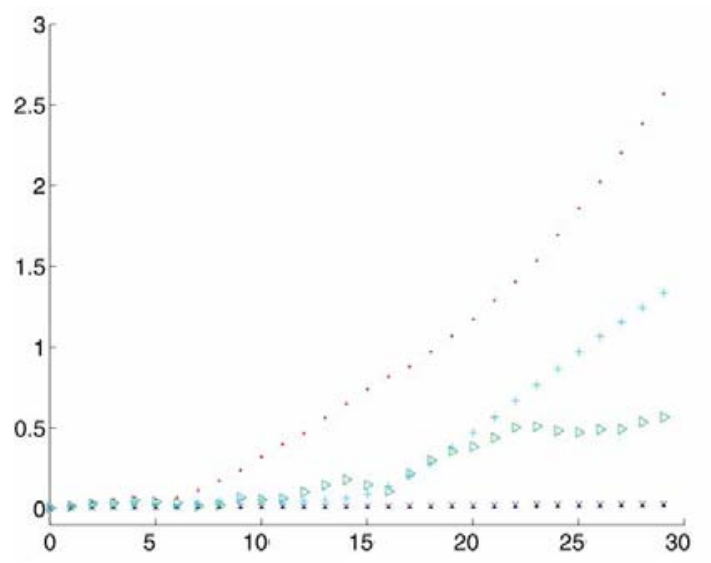

Fig. 10. The error for the estimated position vs the distance travelled by each robot when the estimation is carried out with only odometry (red .), fusion of odometry with the relative distance (green $\triangleright$ ), fusion of odometry with the relative orientation (sky-blue + ), fusion of odometry with the relative bearing (blue $\times$ ) and fusion of odometry with the three relative observations together (black .). The plotted error is averaged over the seven robots.

The proposed approach extends the method introduced in [14] by considering the most generic relative observation between two robots. The equations for this generic relative observation were firstly provided and then specialized for the cases of the relative bearing, the relative distance and the relative orientation. The results from several simulated experiments were also provided. Our first conclusion is that the relative bearing seems to better integrate with the odometry and it is able to reduce the error on the pose estimate better than for the other relative observations. In particular, the improvement is much higher (at least one order of magnitude) than the one achievable by using the other two relative observations (relative distance and orientation) whenever the robot trajectories are similar as the ones presented on figure 2 .

We are currently implementing this strategy on real platforms in our laboratory. We are using a webcam as the exteroceptive sensor and we extract the relative bearing from the images. We are also generalizing this approach by considering the following extensions:

- Integrate the approach presented in this paper with the strategy introduced in [11] for simultaneously localizing and calibrating an odometry system. According to this strategy each robot will be characterized by six parameters instead of three, since the parameters characterizing the systematic odometry error will also be included in the state estimated through the filter (the radii of the two wheels and the distance between them).

- Combine this approach with the control strategy introduced in [6] in order to maximize the number of relative observations during the navigation.

- Consider relative observations among more than two robots. This means that the function $h$ in (8) will depend on more than two robot configurations.

Finally, we are performing a theoretical observability anal- 
ysis to evaluate the information content of each considered relative observation.

\section{APPENDIX}

\section{DERIVATION OF EQUATIONS (9-10)}

We want to derive the update equations for the state $X$ in (1) and its covariance matrix in (2) when the information coming from an observation characterized by the equation (8) is integrated through an Extended Kalman Filter $(E K F)$. The observation described by (8) is a special case of the following observation on the entire state:

$$
z=\tilde{h}(X)+w
$$

Let us indicate the estimation for the state $X$ and its covariance matrix before integrating this observation by $\hat{X}$ and $P$. By applying the standard equations of the $E K F$ [2] we get:

$$
\hat{X}_{n e w}=\hat{X}+P H^{T}\left[H P H^{T}+R\right]^{-1}[z-h(\hat{X})]
$$

and

$$
P_{\text {new }}=P-P H^{T}\left[H P H^{T}+R\right]^{-1} H P
$$

where $R$ is the covariance of $w$ in (A.1) and $H$ the Jacobian of the function $\widetilde{h}$ with respect to state $X$ computed in $\hat{X}$.

Now, when $\widetilde{h}$ characterizes a relative observation between the robots $R_{i}$ and $R_{j}$ it can be characterized through the function $h$ in (8). It is easy to verify that, in this case, the previous Jacobian $H$ is:

$$
H=\left[0, \ldots, 0, H_{i}, 0, \ldots, 0, H_{j}, 0, \ldots, 0\right]
$$

where $H_{i}$ and $H_{j}$ are respectively the Jacobian of the function $h$ with respect to $X_{i}$ and $X_{j}$ computed in $\left(\hat{X}_{i}, \hat{X}_{j}\right)$. From (2) and (A.4) it is now easy to obtain:

$$
\begin{gathered}
P_{z z}=H P H^{T}+R= \\
=H_{i} P_{i i} H_{i}^{T}+H_{i} P_{i j} H_{j}^{T}+H_{j} P_{j i} H_{i}^{T}+H_{j} P_{j j} H_{j}^{T}+R
\end{gathered}
$$

and finally, from (A.2) and (A.4) by considering the robot $R_{l}$ it is possible to get the equation (9) and from (A.3) and (A.4) we obtain for the block $P_{l f}$ the equation (10).

\section{Acknowledgments}

This work has been supported by the European project RECSYS (Real-Time Embedded Control of Mobile Systems with Distributed Sensing) and by the Swiss National Science Foundation No. 200021 - 101886.

\section{REFERENCES}

[1] K.O.Arras, N.Tomatis, B.T.Jensen and R.Siegwart, "Multisensor on-thefly localization: Precision and reliability for applications", Robotics and Autonomous Systems 34, pp. 131-143, 2001.

[2] Y.Bar-Shalom, T.E.Fortmann,, "Tracking and data association, mathematics in science and engineering", Vol 179, Academic Press, New York, 1988.

[3] Chenavier F. and Crowley, J.L., (1992), Position Estimation for a Mobile Robot using Vision and Odometry. IEEE International Conference on Robotics and Automation (ICRA), Nice, France, 1992

[4] Chong K.S., Kleeman L., "Accurate Odometry and Error Modelling for a Mobile Robot," International Conference on Robotics and Automation, vol. 4, pp. 2783-2788, 1997.

[5] Crowley, J.L., (1989). World Modeling and Position Estimation for a Mobile Robot Using Ultrasonic Ranging. IEEE International Conference on Robotics and Automation (ICRA), Scottsdale, AZ, 1989.

[6] S.I. Magnus Egerstedt and Xiaoming Hu, 2001, Formation Constrained Multi-Agent Control, IEEE Transaction On Robotics And Automation Vol 17, No.6, December 2001

[7] D. Fox, W. Burgard, H. Kruppa, S. Thrun, 2000, A Probabilistic Approach to Collaborative Multi-Robot Localization, Autonomous Robots $8,325-344,2000$

[8] R. Grabowski, L.E. Navarro-Serment, C.J.J. Paredis, P.K. Khosla, 2000, Heterogeneous Teams of Modular Robots for Mapping and Exploration, Autonomous Robots, Vol. 8, no. 3, 293-308, June 200

[9] Howard A., Mataric M.J. and Sukhatme G.S., "Localization for Mobile Robot Teams Using Maximum Likelihood Estimation", International Conference on Inteligent Robot and Systems (IROSO2), Volume: 3 , 30 Sept.-5 Oct. 2002 Pages:2849 - 2854, Lausanne.

[10] K. Kato, H. Ishiguro, M. Barth, "Identifying and Localizing Robots in a Multi-Robot System Environment" International Conference on Inteligent Robot and Systems (IROS99) 1999

[11] Martinelli A, Tomatis N, Tapus A. and Siegwart R., "Simultaneous Localization and Odometry Calibration" International Conference on Inteligent Robot and Systems (IROSO3) Las Vegas, USA

[12] J. Neira, J.D. Tardos, J. Horn, G. Schmidt, Fusing range and intensity images for mobile robot localization, IEEE Transaction On Robotics And Automation Vol 15, No.1, 1999.

[13] Rekleitis, I.M., Dudek, G., Milios, E.E., "Multi-robot cooperative localization: a study of trade-offs between efficiency and accuracy" International Conference on Inteligent Robot and Systems (IROSO2) Lausanne, Switzerland

[14] S.I. Roumeliotis and G.A. Bekey, 2002, Distributed Multirobot Localization, IEEE Transaction On Robotics And Automation Vol 18, No.5, October 2002

[15] J.R. Spletzer and C.J. Taylor, "A Bounded Uncertainty Approach to Multi-Robot Localization" International Conference on Inteligent Robot and Systems (IROSO3) Las Vegas, USA, 2003 